\title{
Breaking the Curse of Visual Analytics: Accommodating Virtual Reality in the Visualization Pipeline
}

\author{
Matthias Kraus $^{(凶)}$, Matthias Miller, Juri Buchmüller, Manuel Stein, \\ Niklas Weiler, Daniel A. Keim, and Mennatallah El-Assady \\ University of Konstanz, Konstanz, Germany \\ \{matthias.kraus, matthias.miller, juri.buchmueller, manuel.stein, \\ niklas.weiler, daniel.keim,mennatallah.el-assady\}@uni-konstanz.de \\ https://www.vis.uni-konstanz.de/
}

\begin{abstract}
Previous research has exposed the discrepancy between the subject of analysis (real world) and the actual data on which the analysis is performed (data world) as a critical weak spot in visual analysis pipelines. In this paper, we demonstrate how Virtual Reality (VR) can help to verify the correspondence of both worlds in the context of Information Visualization (InfoVis) and Visual Analytics (VA). Immersion allows the analyst to dive into the data world and collate it to familiar real-world scenarios. If the data world lacks crucial dimensions, then these are also missing in created virtual environments, which may draw the analyst's attention to inconsistencies between the database and the subject of analysis. When situating VR in a generic visualization pipeline, we can confirm its basic equality compared to other mediums as well as possible benefits. To overcome the guarded stance of VR in InfoVis and VA, we present a structured analysis of arguments, exhibiting the circumstances that make VR a viable medium for visualizations. As a further contribution, we discuss how VR can aid in minimizing the gap between the data world and the real world and present a use case that demonstrates two solution approaches. Finally, we report on initial expert feedback attesting the applicability of our approach in a real-world scenario for crime scene investigation.
\end{abstract}

Keywords: Visual analytics $\cdot$ Virtual reality $\cdot$ Visualization theory

\section{Introduction}

Nowadays, data is collected at any time and in any context, in order to gain knowledge about real-world circumstances. Collecting data means storing

This work has received funding from the European Unions Horizon 2020 research and innovation programme under grant agreement No 740754 and from the project FLORIDA (project number 13N14253) by the German Federal Ministry of Education and Research (BMBF, Germany) and the Austrian Security Research Programme KIRAS owned by the Austrian Federal Ministry for Transport, Innovation and Technology (BMVIT). 
digitized snapshots that reflect properties of the real world at a specific point in time. In this article as well as in previous work [42], we use the term data world to refer to the sum of related snapshots collected for a particular use case. To eventually gain knowledge from the collected data, visual data exploration approaches, executed on the data world, are mostly indispensable due to the constantly growing amount of data. However, the snapshots contained in the data world never completely reflect reality, but rather a small fraction of the actual real world that lacks contextual information. Crucial context information can quickly be overlooked, which may lead to false conclusions about the real world. Hence, any analysis carried out on the basis of the data world provides results that are at most applicable to the data world itself and not to the real world as initially intended.

In previous work [42], we raised awareness of this curse of visual data exploration (see also Sect.3) and proposed two potential strategies which help to identify and eliminate differences between the data world and the real world. The first strategy revolves around projecting analysis results back into the real world to confirm these results. Based on the projection of analysis results into a space which is closer to the real world, the user can consider additional contextual information to reveal contradictions that might otherwise stay unnoticed. The second strategy reconstructs the real world from the data contained in the data world to verify its validity. The reconstruction is intended to consider all relevant aspects of the real world for the analysis. This enables the user to compare the reconstruction with the real world to uncover differences such as missing features. For each of the two strategies, we provided a concise proof of concept.

In this article, we build on this existing foundation and work out in detail how Virtual Reality (VR) will help us to bridge the gap between the data world and the real world in the future. In the recent past, VR applications have already gained in importance, in particular in the computer graphics sector. For the information visualization and visual analytics domain, several approaches have been proposed as well $[33,55,73]$, but the potential of virtual reality has not yet been fully explored and established. To show that virtual reality is helpful for problems that occur within visual data exploration, we must prove that virtual reality has a place in the established information visualization pipeline $[14,24,35]$, too (Sect.4). Accordingly, in Sect.4, we introduce our concept of virtual reality for information visualization and emphasize that VR is not inferior to conventional mediums (printouts, screen-based displays) when applied correctly. Instead, we argue that it is possible to obtain visualizations in VR that are as suitable as those displayed using conventional mediums. Moreover, we show that specific properties inherent to virtual reality environments (VREs) can be used to extend existing visualizations or even to establish new ones. After laying this foundation and defining the place of VR in the information visualization pipeline, we demonstrate in Sect. 5 which advantages (Sect. 5.1) VR has to minimize the effects (Sect. 5.2) or even to solve the initially described curse of visual data exploration. Ultimately, in Sect. 6, we describe an extensive real-world use case in crime scene investigation, applying both of the aforementioned strategies 
to break the curse. Overall, our contribution consists in (a) identifying dimensions to compare visualizations displayed on different mediums, (b) providing an overview of currently existing major drawbacks of VR for data visualization, (c) listing conceivable present and future possibilities of VR, and (d) discussing potential benefits associated with the use of VR to minimize the gap between the real world and the data world.

\section{Background and Related Work}

In this section, we deduce the key terms used throughout the article from related literature. For many expressions, such as VR, immersion, or presence, numerous different definitions exist. Our literature review aims at eliminating ambiguities by defining the terminology, thus paving the way for later lines of argumentation. In addition, several examples of $\mathrm{VR}$ visualizations are given, which show positive effects of VR in the context of InfoVis to demonstrate the potential of VR and to motivate its application.

\subsection{Virtual Reality and VREs}

According to the dictionary, we can define the terms 'virtual' and 'reality' as follows: Virtual is something that is "temporarily simulated or extended by computer software" (e.g., virtual memory on a hard disk) [21]; reality is "something that exists independently of ideas concerning it" [20]. To compare VR as it is used in computer science with conventional mediums, we first need to specify what exactly is meant by a VR system in this context. The first experiments with VR technology were done several decades ago [30]. Since then, a vast amount of different VR equipment has been developed, ranging from pocket-sized cardboards like the Google Cardboard [32] to room-sized installations like the CAVE [16]. Different properties of VR prototypes may be the reason why several definitions have been introduced in recent years. Although most of them are relatively similar, they differ in one crucial aspect: They describe the defining properties of VR from different perspectives. We were able to identify three general types of definitions for virtual reality.

* Hardware-centered VR. Some VR definitions focus mainly on the hardware aspect. They usually include some kind of stereoscopic display as well as interaction controllers or data gloves. A representative definition of this category was introduced by Ellis [26], who links virtual reality with the hardware it is created with - a head-mounted display. VR displays track users' head positions and adapt themselves accordingly, enabling users to navigate through the virtual reality environment. VR is created by VR hardware.

- Human-centered VR. Latta and Oberg [45] provide an example of what we call a human-centered definition of virtual reality. They define virtual reality as an interplay of hardware and user. The hardware monitors human behavior and stimulates the human perceptual and muscle system in return. 
Their model of a VR system consists of effectors and sensors. Effectors describe the hardware that the VR system uses to stimulate the human body while the sensors are responsible for detecting human actions. VR is created by the synergy of human and computer.

: Concept-centered VR. Concept-based approaches deduce virtual reality from a conceptional construct. For instance, Steuer [62] derives VR from the concept of telepresence. With regard to Steuer, someone is located in a virtual reality if he or she is telepresent in that environment. He defines telepresence as presence originating from a communication medium (such as a VR headset). Moreover, the degree of the telepresence (and therefore immersion) depends on two dimensions: vividness and interactivity. Vividness describes how well the virtual world emulates the real world, and interactivity describes how much the user can influence it. VR is created by applying a concept to the user (e.g., telepresence: mentally transporting users to a different place).

\subsection{Immersion and Presence}

Each of these VR definitions addresses, explicitly or implicitly, the two key aspects of VR: immersion and presence. They describe states of a user located in a virtual reality environment. Even though both terms are key elements of VREs, there is an ongoing discussion about their meaning and definition. For example, Cummings and Bailenson summarize the concept of presence as the feeling of "being there" in a mediated virtual environment. Presence is responsible for the effectiveness of VR applications as it magnifies stimuli and virtual interactions (2 human-centered) [17]. McGloin et al. state that the term 'immersion' is synonymous with presence [49] and offer the same definition as Cummings and Bailenson.

According to Slater and Wilbur, the term 'immersion' describes the employed technological basis for the implementation of a VRE that influences its medial quality (hardware-centered) [60]. Immersion comprises the ability of a system to provide persuasive conditions that are perceived through human sensory organs. Consequently, the degree of 'immersion' of a system is an objective measure which can be used to compare the quality of different systems. Immersion depends on the ability of a system to fade out the physical reality by addressing all senses of an immersed person as good as possible. The richness of provided information eventually affects the vividness of the VRE ( concept-centered) as a function of screen resolution and fidelity. Besides, self-perception is a further crucial aspect of immersion, since having a virtual body in a VRE creates the perception of being part of the constituted surrounding.

Witmer et al. describe presence as the subjective experience of being transported to another place or environment ( concept-centered) [72]. The intensity of presence influences the sensation of an individual experiencing the VRE instead of the actual environment. Ijsselsteijn and Riva consider the experienced 
presence to be a complex and multi-dimensional perception shaped by various cognitive processes and the composition of sensory information humancentered) [40]. The perceived degree of presence depends on the ability of the immersed person to have control over the mediated information. The simultaneous stimulation of multiple sensors affects cognition, perception, and emotions, which are key components of presence. The better these dimensions are addressed by the VR system, the higher the degree of presence that can be experienced in a VRE.

According to this extensive amount of recent work, optimizing presence is the primary objective when designing a VRE. Since presence directly depends on the degree of immersion, technological improvements that enhance perceived immersion (e.g., an increase in resolution) also augment presence [18]. High degrees of immersion allow users to have an improved psychological experience of "being there" [17]. The sensation of presence in a VRE can significantly vary between persons as it depends on individual factors such as attentional resources and physical conditions.

\subsection{VR for Information Visualization in VA}

In the past, various applications for VR have been developed. Some of them show benefits, among others, in the educational [34], biochemical [55], and physiological $[5,37,69]$ domain. But also in the context of InfoVis, various VR applications have proven to be useful, particularly for (geo-)spatial data, volume data, and abstract multivariate data. For example, Gruchalla [33] demonstrated a beneficial effect of immersion on well-path editing tasks for oil rigs. Zhang et al. [73] were able to show that VR can help to improve the understandability of geometric models.

Attempts were also made to visualize abstract data, i.e., data that has no natural representation in the real world, in VR. For instance, Erra et al. [27] found a beneficial effect of VR on graph exploration tasks. Donalek et al. even claim that VR "leads to a demonstrably better perception of a datascape geometry, more intuitive data understanding, and better retention of the perceived relationships in the data" [23]. They presented and compared different VR systems for the immersive visualization of abstract data (e.g., scatterplots). Merino et al. [50] compared different mediums for 3D software visualizations and found that immersive $3 \mathrm{D}$ environments led to better recollection rates in experimental subjects than standard computer screens and physical 3D-printed representations. Wagner Filho et al. [68] investigated the performance of users in a variety of analysis tasks using a virtual desk metaphor and a traditional desktop environment, respectively. They found equal or lower error rates in perception tasks with slightly poorer time efficiency, but also a higher perceived efficiency of their proposed VRE. 


\subsection{Uncertainty and Validation}

When analyzing data, uncertainties must always be taken into account. Uncertainties can typically occur both in the data to be analyzed (for example, when collecting data) and in the processing and displaying approaches used (for example, when aggregating data). Uncertainty factors inherent to virtual reality environments are largely caused by the latter since VREs as display technology do not interfere with the displayed data. Sources of uncertainty in VREs mostly impact a user's perception. For example, the resolution of many VR headsets is usually lower than that of current two-dimensional displays. To capture the range of uncertainties that may affect users of a VRE during a data analysis process, a generalized definition should be applied. MacEachren et al. [47] identified different components into which uncertainties can be divided. Another, more hierarchical approach is illustrated by Skeels et al. [59], who describe three different abstraction levels for uncertainty in various domains, including Information Visualization. The most basic level, measurement precision, refers to imprecisions in data gathering, for example in positional sensors of VR equipment. The other two levels, completeness and inferences, describe sources of uncertainty due to a loss of information in sampling or projection processes as well as in predictions of future unknown values. These three levels are completed by an additional credibility layer which covers the trustfulness of a data source. Specifically with regard to the visualization domain, Gershon et al. [31] apply the concept of an imperfect world to imperfections in data collection as well as visualizations themselves (e.g., overplotting issues).

In its original context, the concept of measurement precision as mentioned previously typically applies to the accuracy of the gathered data which are to be explored or analyzed. However, in VR, sensors are employed to translate a user's movements into reciprocal perspective changes to create the effect of immersion. Inaccuracies of these sensors lead to a deviation between a user's expectation with respect to the change in the field of view according to his movements and the actual visual output of the VRE system. If this deviation is too large, for example, due to high latency, negative effects on a user's immersion and engagement can occur. In addition, such sensory deviations are one of the main causes of motion sickness in VR environments. Niehorster et al. [51] conducted an in-depth analysis of sensor accuracy in a state-of-the-art VR product and concluded that, while deviations are present, current off-the-shelf technologies are sufficient for many scientific requirements.

While the accuracy of a system in terms of translating physical movements into virtual movements is critical for the usefulness of a VRE, many use cases also incorporate physical objects into the VRE. For example, physical walls or other obstacles need to be visually communicated to a user. Potential uncertainties in the mapping of physical objects and virtual representations form a second type of sensor-related uncertainty issues for VREs that must be considered. For example, Kim [41] provides a computer-vision based approach for the calibration of VREs with the physical space. Still, not all uncertainties can be countered or completely compensated. Consequently, coping mechanisms need to be applied 
to diminish the mentioned effects. Benford et al. [3] offer various mechanisms for this. For example, positional errors can be countered with additional position validation. If this is not sufficient, audible cues can improve users' orientation. Another proposed approach is to actively confront the user with the existing uncertainties so that the user is able to create a mental model of the uncertainties experienced and to face them in the future with the aid of experience.

In general, and independently of the visualization medium, a data validation process can be used to verify the plausibility of data values and to counter uncertainty issues, as explained by the United Nations Statistical Commission and Economic glossary [65]. A more detailed view on validation is provided by Wills and Roecker [71], who interprete validation as the ability of a model to deal with variances, missing values, and outliers in a dataset. In recent years, different techniques for dealing with outliers and anomalies have been researched, including the detection of anomalies [11], event detection in time series datasets [22], and noise detection [57]. A survey on further outlier detection techniques is provided by Hodge and Austin [36], identifying three approaches, including pure statistical distribution analysis, labeled-based classifier training on all available data points, and label-based training solely on known outliers.

\subsection{Positioning of Our Work}

Now that we have surveyed different types of definitions for virtual reality and immersion, we first elaborate on the generic problem we call the "curse of visual data exploration" by recapitulating previous work and bringing it into the current context. Subsequently, we position generic information visualization in a visual analytics workflow model. By deploying a theoretical, ideal VR environment as a substitute for a conventional medium (e.g., monitor screen), we can show its theoretical equality. Then, we reduce the requirements of the ideal VRE and adjust its properties to a more viable VRE. We then discuss where it still performs equally to a monitor screen (Sect.4). Afterward, we point out benefits of VREs compared to conventional mediums by addressing each step of the visualization pipeline (Sect.5). We will then deploy a use case with initial expert feedback to demonstrate how VR could help bridge the gap between the data world and the real world (the curse of visual data exploration).

\section{The Curse of Visual Data Exploration}

Visual Analytics is commonly used to gain knowledge from large datasets which have been recorded from the real world. Consequently, the resulting knowledge is considered applicable to the real world. However, the gathered data represent only a small fraction of the actual real world and lack contextual information. It is possible that crucial context information is not included in the analysis process, leading to false conclusions about the real world. As a consequence, resulting findings relate to the world represented by the collected data and may not be valid for the real world. In previous work [42], we raised awareness of this 
discrepancy between the data world and the real world (The Curse of Visual Data Exploration), which has a large impact on the validity of analysis results. In the following, we will summarize the main characteristics of the curse of visual data exploration and describe two strategies to break the curse, based on our previous work [42]. We then build on this existing foundation incorporating a thought experiment and work out in detail how Virtual Reality will help us to bridge the gap between data world and real world in the future.

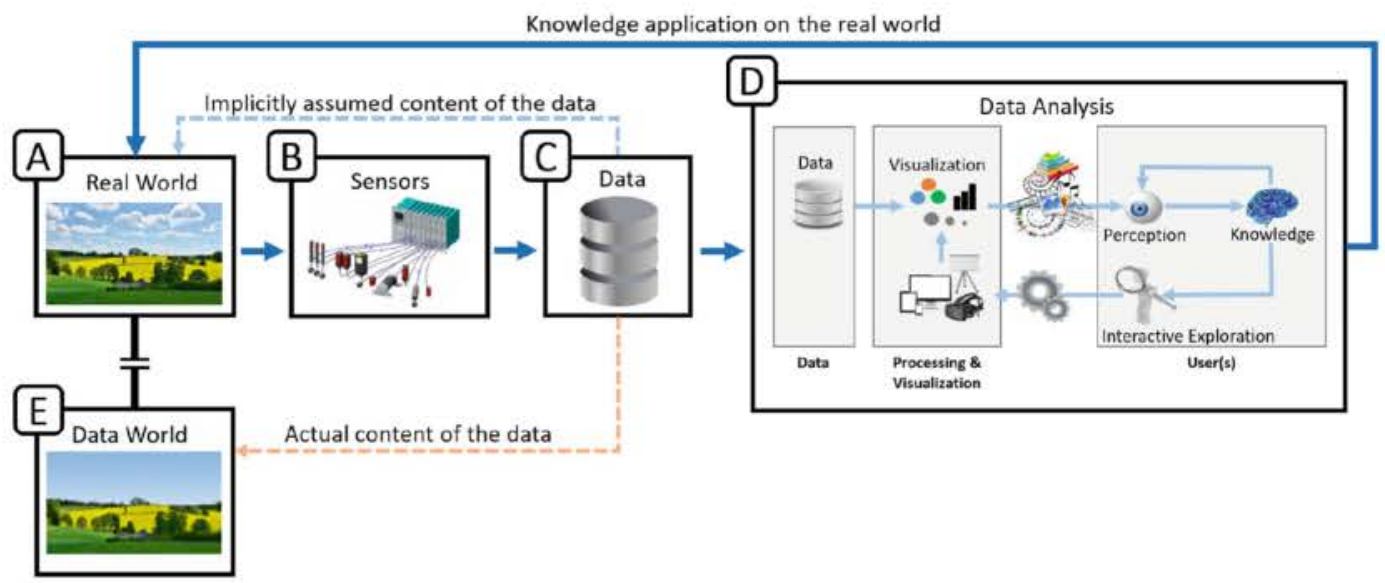

Fig. 1. The curse of visual data exploration displayed in an extended visual analytics model. A typical visual analytics task starts in the real world (A). The goal is to gain information about real-world properties. Information is collected using sensor technology (B) and stored in a database (C). Subsequently, the gathered data is processed in a VA pipeline. This process is illustrated in (D) as an adoption of the VA model proposed by van Wijk et al. [70]. Knowledge generated in such workflows is assumed to apply to the real world, implying that the collected data is a complete and correct copy of the real world. However, the data collected on which the analysis is based contains only a fraction of real-world aspects (the data world (E)). This can have a critical influence on the analysis process as the generated knowledge may not be complete or even invalid in the real world. Figure adopted from [42].

Visual Analytics Pipeline. Figure1(A-D) depicts the overall visual analysis workflow. An analyst strives to gain knowledge about a particular real-world scenario (A). In order to perform an analysis, data from the real world must be collected, which can be achieved, for example, by using sensors (B). The digitized data is stored (C) to be subsequently analyzed in a VA workflow (D). Knowledge gained from the procedure is applied to the investigated real-world scenario (A).

The Curse. However, the digitized data used for the analysis do not flawlessly represent the real world. It is almost impossible to guarantee that the information used for the analysis is exclusive, complete, and correct. Thus, we claim that data is always wrong to some extent [42]. As shown in Fig. 1(A, E), we differentiate between the real world that is investigated and the data world, which is the 
entirety of the collected data based on which the actual data analysis is carried out. Insights gained during visual analysis procedures (D) therefore apply primarily to the data world. If the real world and the data world differ in essential aspects, the knowledge applicable to the data world may not be transferable to the real world as it leads to wrong assertions about properties of the real world. We call the discrepancy between the real world and the data world the curse of (visual) data exploration.

Breaking the Curse. We proposed two strategies to break the curse [42]. Both strategies aim to provide additional context information and draw the analyst's attention to potentially neglected dimensions in the analysis. The first approach consists in reconstructing the real world as far as possible from the data world so that the analyst can verify its validity. For example, when analyzing the collective behavior of birds, models of birds can be animated in a virtual environment which reflects the original scenario as far as possible, by incorporating context that was not necessarily included in the analysis itself such as the landscape or weather conditions. The second approach is to project analysis results into the real world or a less abstract reflection of the real world. This could be achieved, for instance, by projecting soccer analysis results into the original camera footage of the soccer match. By projecting the analysis results into a space that is closer to the real world, analysts can identify contradictions that would not be noticed otherwise.

Thought Experiment: Hypothetical, Ideal $V R$. In this paper, we investigate how and to what extent the discrepancy between the data world and the real world (see Fig. 1A, D) can be minimized by deploying VR and the first strategy (reconstructing the real world). As previously stated [42], the gap would be minimal if the data world used for the analysis perfectly resembled the real world. Therefore, we need to develop a theoretical framework to discuss this important topic at a hypothetical level where the data world perfectly resembles the real world and is perceived in a VRE. By doing so, we first assume a VRE that perfectly resembles the real world (1) and elaborate on accompanied advantages (2).

1. What if a VRE is Actually Perceived Like Reality? At least in theory, it is possible to see VR as an equivalent medium for perceiving visualizations. Consequently, we can imagine a hypothetical VR environment that perfectly reflects a real environment, for example, a so-called "Substitutional Reality" environment $[58,64]$ that replaces each real object with a virtual representation. In our case, instead of substituting each real object with an alternative virtual object with similar properties, it is replaced by a virtual representation of itself. For instance, a room with furniture should in our case in VR be represented exactly as it is in the real world. All objects are positioned where they are in the real world. As a result, anyone entering the "duplicated world" in this example would physically walk through the real room and perceive the virtual room (which looks like the real room). Any cloned, "virtual" object could be implicitly touched and interacted with as the virtual world can be considered a "duplicate" of the real environment and each virtual object has a real counterpart. 
2. What are the Advantages of Such a Possibility? As soon as we have constructed a perfect representation of the real world, we need to illustrate why we should not simply make use of a conventional medium (such as a regular monitor screen) to inspect the replication of the real world, instead of a VRE. As a first step, we show in Sect. 4 that VR has a place in the established information visualization pipeline. In Sect. 4.1 we demonstrate how the ideal VR can be embedded in the information visualization pipeline, while in Sect. 4.2 we show how perfect a reconstruction of the real world must be in order to be perceived and considered as equivalent by a user. We use the resulting findings in Sect. 5 to verify whether VR is actually preferable to a conventional screen in our scenario.

\section{VR in the Visualization Pipeline}

In this section, we present a hypothetical, ideal VR as part of the previously presented thought experiment and demonstrate the equality between the hereby created VRE and a conventional screen-based setup with regard to the visualization pipeline (Subsect.4.1). Furthermore, we discuss to what extent all prerequisites and assumptions made during the thought experiment are necessary to maintain equality between the compared mediums (Subsect.4.2).

\subsection{Embedding the Ideal VR in the InfoVis Pipeline}

Since we are mainly concerned with implications and effects on InfoVis and VA procedures, we examine properties of seven dimensions that constitute the main characteristics of a visualization pipeline. As illustrated in Fig. 2, we adopted the VA pipeline by van Wijk et al. [70]. We consider their model to be particularly suitable for our needs, as they provide a highly generalizable pipeline with the focus on the "user side" (knowledge generation, interaction, perception, etc.) instead of the "computer side" (parameterization, model evaluation, etc.). Therefore, we use the states and transitions they identified in the pipeline to compare two different mediums that display the same visualization. We compare the most established medium used for InfoVis and VA tasks - the monitor screen - with the ideal "VR-display" described in the previous section.

(i) Data. Input of the information visualization pipeline (Fig.2(i)). Assuming that the data collection process is not a visualization task itself, this dimension is not directly influenced by the medium used. However, some data types are predestinated for specific visualizations, and their performance could be affected by the medium used.

(ii) Visualization. Data transformation and visual mapping steps, as they are described by Card et al. [10] in their well-established reference model for visualization, are covered by this dimension (Fig. 2(ii)). It describes how the specification is applied to the data (e.g., how the data is transformed, how visual variables are mapped). The outcome is an "Image" that can be perceived by 


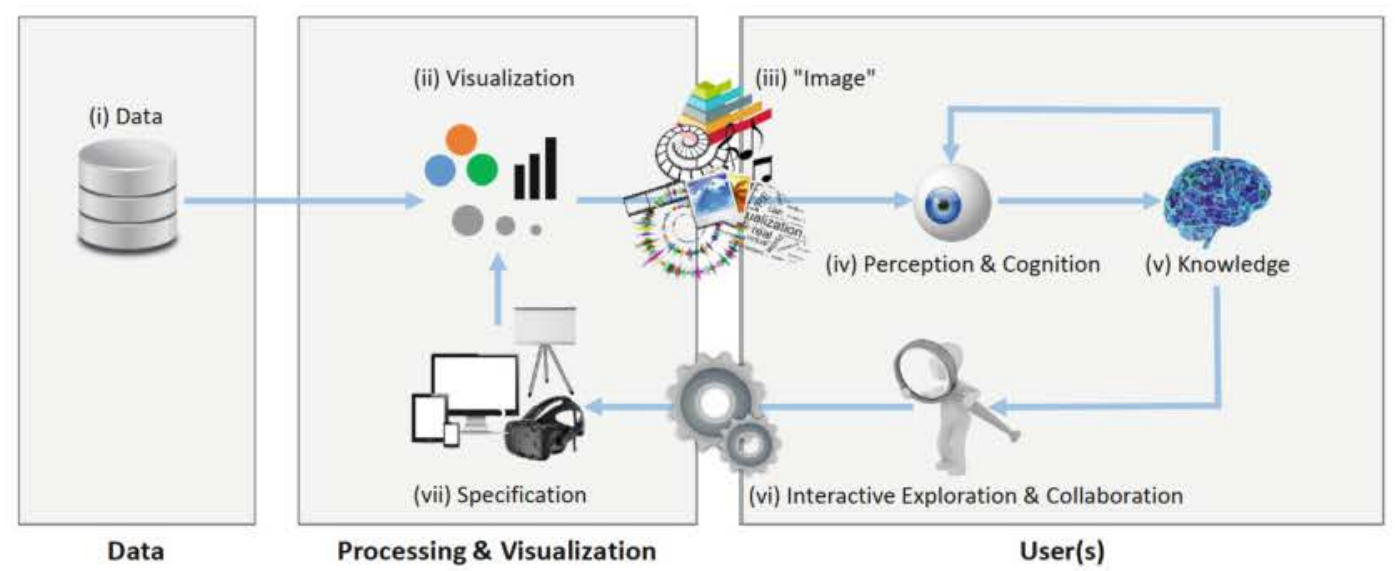

Fig. 2. Visualization pipeline adopted from van Wijk et al. [70]. Data (i) is transformed and mapped (ii) based on specifications (vii). A resulting "Image" (iii) of the visualization is perceived (iv) by the analyst who can apply prior knowledge (v) to interpret the visualization and to acquire new knowledge (v). Subsequent interactive exploration (vi), possibly through collaboration (vi), may lead to the adaption (vi) of the specification (vii).

the user. The visualization is displayed on the virtual monitor in the VRE - just exactly the same way as it is shown on the real monitor. Any data transformation, visual mapping, etc. applicable to the "real" visualization on the monitor screen would also be applicable to the VR visualization.

(iii) "Image" (Image, Animation, etc.). Outcome of the visualization step (Fig. 2(iii)). This dimension describes properties of the actually perceptible entity that is observed by the user. In the field of information visualization, this is most often a 2D image, but it could also be audio, video or haptic feedback, or any other perceivable signal. The process of transforming the visualization into a user-perceivable signal ("Image") constitutes the view transformation step in the model by Card et al. [10]. Applicable types of "Images" depend on the specification given in a respective analysis task. Vice versa, hardware specifications, such as the medium used for display, define characteristics of the "Image". Assuming that the VRE is ideal, the image presented to the user is the same as in the real world.

(iv) Perception $\&$ Cognition. Conceiving the "Image" (Fig. 2(iv)). This dimension describes how the visualization is perceived and processed by the user. This step is user-, visualization- and specification-dependent. Different users may perceive the "Image" differently, for instance, due to diverse personal notions (e.g., interpretation of "Image") or physical differences (e.g., color perception). Obviously, also the type of "Image" (e.g., line-chart visualization) and its specification (boundary conditions such as the hardware used for display), influence its perception and cognition. In an ideal VRE, limitations like low resolution, latency, or the weight of a head-mounted display are neglected. In this case, the VRE and 
the real environment cannot be visually distinguished from each other. Therefore, perception and cognition in the VRE would on all channels be equal to a real-world experience.

(v) Knowledge. Outcome of the visualization pipeline: the knowledge gained in the course of the analysis (Fig. 2(v)). Its volume and character depend, among other things, on prior knowledge (e.g., experience, domain-specific knowledge) and the expressiveness of the inspected visualization. The latter depends on several properties, such as the characteristics of the underlying data, the type of visualization used, and the aspect of interest. In an ideal VR, everything is cloned perfectly, leading to the same situation in the virtual and real environment. Therefore, perceiving a visualization in the ideal VRE instead of inspecting it on a computer screen would not affect the knowledge generation process in any way.

(vi) Interactive Exploration $\&$ Collaboration. Types of interaction and their properties (Fig. 2(vi)). This dimension describes where and how users can interact with the system (i.e., adaption of specification) or with other users (collaboration). In our thought experiment, the real world is cloned, and everything in the virtual copy can be touched and interacted with. Interaction would be the same as in the real world. Collaboration could be achieved, for example, by screen sharing (of the virtual monitor screen).

(vii) Specification. Boundary conditions for the creation of the visualization (Fig. 2(vii)). The specification defines properties like the type of visualization, the dataset to be considered or the mapping of data attributes to visual variables. Moreover, this dimension describes the hardware and (physical/virtual) environmental conditions. Data processing is covered by this dimension as well: Preprocessing, such as normalization or data cleansing, as well as actual data mining steps, such as clustering, pattern detection or classification are implicitly reflected in the pipeline. Such operations can be seen as the execution of an adaption of the specification that leads to a manipulated dataset. For instance, in ML/VA workflows, the specification includes any parameterization for the training of the ML model (learning rates, activation functions, etc.). The specification can be adapted by the user through interaction. Most aspects of this dimension are independent of the medium used for the final visualization. Even though the hardware on which the visualization is displayed in the VRE is different, the outcome is the same.

\subsection{Renouncing the Ideal}

In this section, we will qualify statements made in Sects. 3 and 4.1 with respect to actual limitations of VREs. Due to technological flaws, the state that a VRE is perceived in exactly the same way as the real world may never be reached. However, for visual analysis, a VRE can already be considered "ideal" if it lacks nothing that would compromise the efficiency or effectiveness of the visualization 
or task. Therefore, the simulation of the real world does not necessarily have to reach a perfect level at which the VRE cannot be distinguished from the real environment.

Starting with the specification, it would never be possible to perfectly replicate a real-world scenario in VR without any flaws. Detail, resolution, photorealistic rendering, real-time monitoring, and cloning of real objects into the VRE are only some examples of the many obstacles. More realistic would be an environment that is anchored at some points to the real environment, but is largely independent. The previous example in the thought experiment could, for example, be reduced to a VRE that only overlaps with the real world in certain parts - the desk and the input devices (mouse and keyboard). Anything else in the VRE could be artificial without directly affecting a particular visual analysis task. Although we would not be able to track remaining physical objects or receive haptic feedback from virtual objects in the VRE, objects would still respond to the input devices used - similar to desktop systems.

The 'workaround' used in the thought experiment to deploy hardware input devices for interaction is not the most obvious. Of course, this may be possible, as several prototypes on the market show (e.g., Logitech [46]), but there may be different interaction possibilities that work better with VR (e.g., the mouse only allows navigation on a plane). Mouse and keyboard are the two most established interaction devices most people are familiar with. Introducing new, VR-optimized devices, such as the Vive controller [38], could result in comparatively high learning effort. However, both types of input devices are possible for use in VA workflows. The main goal is to optimize the visual analysis by finding the most suitable interaction device for a particular task.

Depending on the specification, the generated "Image" would be subject to low resolution, non-photo-realistic rendering, and many other artifacts that affect later perception and cognition. The extent to which these artifacts influence the expressiveness and effectiveness of the presented visualization largely depends on the specification and the visualization, but also the task and the user. Some technological drawbacks can certainly be resolved by future technology (e.g., low resolution, latency, photo-realistic rendering). However, it is highly unlikely that one day, the human brain can be completely deceived. Nevertheless, we argue that perfect delusion is not necessary to achieve an equivalent performance in VR compared to screen-based mediums. The information displayed only needs to be conceived similarly efficient, complete, and exclusive.

\section{Breaking the Curse with Virtual Reality}

After having established in the previous section that VREs can theoretically perform just as well as conventional mediums in terms of conveying information, we attend to the advantages VREs can have. Figure 3 depicts two sides of VREs. The properties discussed in Sect. 4, which compensate conventional mediums, are shown in red. This section focuses on the blue side, presenting exclusive features of VREs that can have a positive impact on different dimensions. Using the 
previous example, one could clone the real environment and interact with it as if one perceived the real world. This would not make any difference to interacting in and with the real world directly, but it would also be possible to do things which are not possible in the real world. For instance, one could manipulate objects in the environment, install "holograms" or 3D visualizations within the room or exclude distracting parts. In the following subsection, we deal with each of the previously determined dimensions (i)-(vii). Subsequently, we discuss various dimensions that are exclusively available in VREs with regard to their ability to aid in breaking the curse of visual data exploration. This means properties of VREs that help to identify data dimensions that were neglected in the visual data analysis process but have implications for the knowledge generation process.
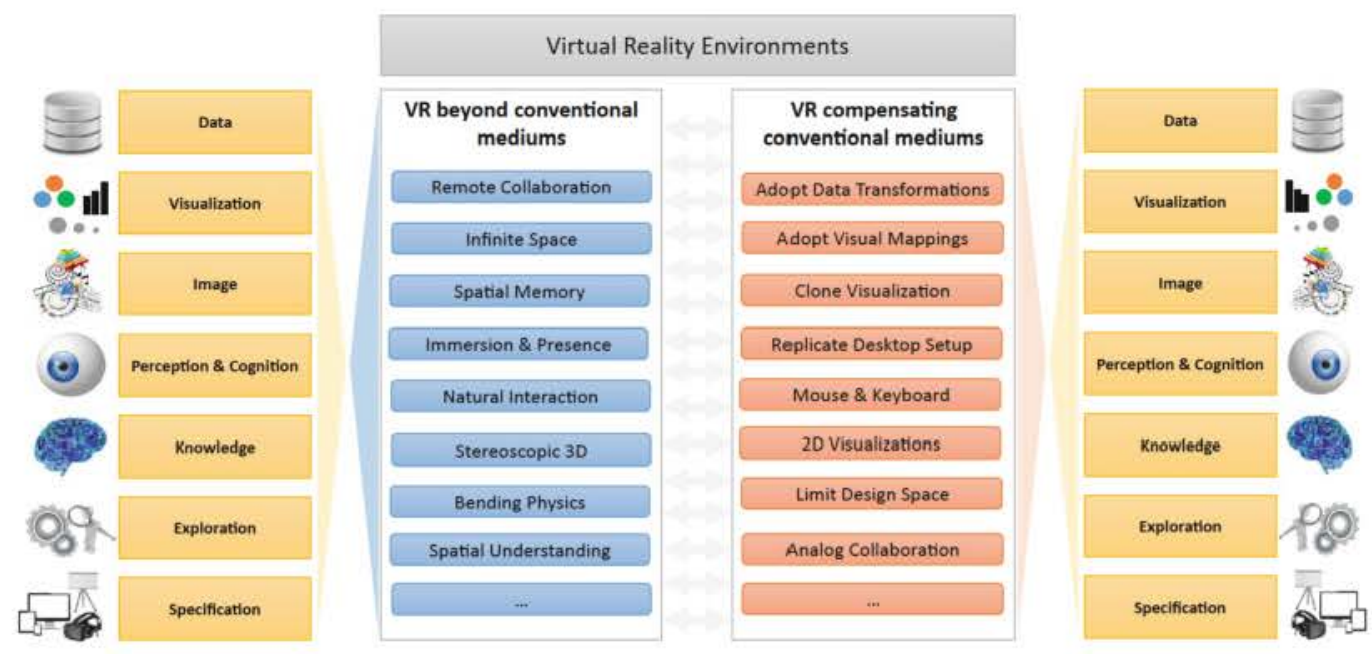

Fig. 3. We investigate if all properties in the visualization pipeline (yellow) for the display of visualizations on a conventional medium (e.g., monitor screen) can be compensated by VREs (right side, red). This is exhaustively discussed in Sect. 4. Red lines indicate to which dimension(s) the examples can be assigned. Subsequently, characteristics of VR that are not present in conventional mediums are discussed in Sect. 5 (left side, blue). Blue lines indicate which dimensions are affected by the exemplary VR characteristics listed. (Color figure online)

\subsection{Implications for Dimensions}

Data (i). The "Data"-dimension is not directly influenced by the medium used for display. Nevertheless, some data (e.g., spatial, 3D, volume) can be displayed more naturally in VR than in other mediums. This means that different data types allow different visualization applications and influence their effectiveness and expressiveness. As shown in the thought experiment, VR cannot only implement any conventional visualization mapping but also extend it by using, for instance, stereoscopic 3D vision. 
Visualization (ii). Previous research has shown that it can be advantageous to use the immersive characteristics of VR to combine multiple views that exploit the three-dimensional aspects of such an environment [67]. The user can work with visualizations in the traditional way, while it is also possible to seamlessly combine both concepts in a single application, lowering the bar for users who are not used to VRE. Thus, the analyst is provided with a flexible system that enables him or her to investigate information as intended. For example, Huang et al. [39] combined GIS and VR in an internet application to support users in decision-making processes by deploying spatial databases and spatial data visualizations. Besides the option to map traditional visualizations of a twodimensional setting into a VRE, it is possible to enrich the presented information with spatial views. In this way, the three-dimensionality of the visualization design space of the VRE can be utilized to integrate inherent topological information with associated spatial information. Based on these findings, VREs enable the usage of more visual mappings and a larger design space compared to monitor-based 2D applications.

"Image" (iii). If we assume that "Image" is the information of the presented visualization that is sensorily perceived by the user, then the number of possible sensory channels increases in a mediated environment. In addition to the twodimensional visualization and potential auditory information, we can add haptic feedback, expand it with stereoscopic images, and even include the sense of smell and taste. For instance, haptic feedback can be implemented through a glove that provides advantageous touch feedback [66]. The perceived "Image" is also improved by the stereoscopic nature of identifying the exact position and depth of an object in a VR scene. The integration of three-dimensional auditory information into a VRE allows guiding through 3D visualizations and improves the intensity of the "Image" [48]. Depending on the application, using audio can be more intuitive than encoding all information visually. Porter et al. have shown how to encode spatial direction information in the sense of smell [53]. A VRE that effectively implements such techniques could subconsciously guide users in a certain direction to find the intended results or support the process of exploration. Theoretically, applying methods that stimulate the sense of taste would widen the "Image" space and substantially enrich the VR experience.

Perception $\&$ Cognition (iv). A major difference to monitor-based visualization approaches is the complete controllability of the environment, which is presented to a user in a VRE. Studies, mainly from the scientific visualization domain, such as the ones conducted by Laha et al. [44,54], indicate positive effects of immersive environments taking advantage of this aspect on the analysis efficiency of users in certain tasks. As a side effect, environmental factors of classic desktop environments such as changing lighting conditions, noise interference, or thirdparty interactions or distractions can be mitigated or even completely prevented. As a consequence, when using VR, a user may be able to focus more on the analytical tasks at hand. 
Knowledge (v). Knowledge application and generation are indirectly influenced by the medium used. For instance, when inspecting a geo-spatial visualization on a conventional screen, the user would perceive a $2 \mathrm{D}$ projection of the scene, whereas he would perceive stereoscopic 3D images when inspecting the same scene in VR. Perceiving the least abstracted copy of the familiar real world (stereoscopic landscape) may help to quickly transfer and apply prior knowledge to the visualization. Vice versa, the less mental mapping is required from the visualization to the real world, the easier it is to transfer gained knowledge to the real world. The smaller distance between the visualization and the real world could ease the transfer of knowledge to the visualization, increase the understandability of visualization results, and thus augment the efficiency and effectiveness of visual analysis procedures. Previous studies (e.g., $[43,50,56])$ have shown that increased immersion can have positive effects on memorability as well. Spatial memory is improved by heightened spatial understanding and muscular memory. In visual analytics tasks, this could enhance efficiency (e.g., cluster identification, analytic provenance) and effectiveness (volume of knowledge extracted).

Interactive Exploration \& Collaboration (vi). Traditionally, one can distinguish between remote collaboration and co-located collaboration. Using VR technology, it is possible to simulate co-located collaboration even though the collaborators are physically separated. This can be realized by projecting the avatars of all collaborators into the same VRE. This approach combines the advantages of both kinds of collaboration: The benefit of remote collaboration of not having to be present in the same room, and the improved interaction possibilities of colocated collaborations. For example, one can point at a specific position to guide other persons' attention. On a monitor, the same can be achieved by the use of a mouse pointer. However, this is limited to pointing at objects shown on the screen itself. Pointing at dashboards, persons or hardware other than the monitor is not possible with the mouse. Another advantage of remote collaboration in VR is that it is possible to see where the other person is looking at. Seeing what collaborators are currently focusing on can help to improve the common conversational grounding.

Specification (vii). In comparison to traditional mediums, one of the main advantages of VR hardware is the fully controllable stereoscopic environment. Visualizations which use a 3D structure to encode information can make good use of this as their shape is easier to identify in a stereoscopic view than in a screenbased 3D view. Compared to other mediums that offer a stereoscopic view, like a 3D print or 3D glasses, VR allows to control and influence the entire visualization space in various ways. This increases the options for displaying information and reduces distractions by removing unnecessary elements from the scene. This works well together with the large field of view that VR hardware usually offers. A large field of view allows showing more information at once since more graphical elements can be seen at the same time. Finally, head-mounted displays are easy to transport, which can be an asset in some cases. Other mediums that 
offer a large field of view, for example, large-scale displays, are often by far more difficult to transport or not transportable at all.

\subsection{Minimizing the Gap Between the Real World and Data World Using VR}

As shown in the introduction of the curse of visual data exploration, it would be optimal for the validity and applicability of analysis results if the discrepancy between the data world and the real world would not exist. In reality, however, this is hardly possible, since the digitization process of real-world properties is already subject to a loss of information. Nevertheless and as previously shown, the use of VR may minimize the gap between the data world and the real world as it allows to inspect the data world more naturally and realistically than on a conventional screen. In VR, the snippet of the real world can be inspected on a lower level of abstraction due to stereoscopic 3D perception, natural navigation, and improved spatial understanding. Because of immersion, differences between the inspected virtual environment and the familiar real world can be identified more easily. For instance, one is automatically aware of the absence of familiar properties, such as the atmosphere, wind, sound, and smell.

For the validation of analysis results, the real world could be reconstructed from the data world as close as possible. Due to an incomplete data world, the reconstruction would suffer from incompleteness as well, calling attention to missing or faulty dimensions in the data. Subsequently, analysis outcomes could be projected into the reconstruction. The analyst could then enter the resemblance of the real world and verify if displayed analysis outcomes logically fit in the displayed environment. For instance, based on experiences from the familiar real world, the user would be able to identify errors in analysis results, such as an outlier in a person's movement trajectory that describes a jump over five meters and back in two seconds. Moreover, the absence of important dimensions that possibly influence the analysis outcome could be identified and included in the next analysis iteration. For instance, in a collective behavior analysis task, tracked animals could be represented in a virtual reconstruction of the landscape in which they actually moved. By inspecting this virtual scenario, the analyst may be able to detect errors in the analysis. For example, analysts may recognize that they have not considered obstacles such as rocks, trees, or rivers that influence the animals' trajectories. Animal paths may have certain shapes due to characteristics of the environment, which are easily overlooked in a basic analysis without a more detailed visual examination. VR could also help to identify dimensions that are more difficult to detect on a screen. Being immersed in a copy of the real world allows the analyst to perceive the environment more naturally. Artifacts that contradict the familiar real-world properties, such as gravity, atmosphere, or three-dimensionality, may be identified more quickly than without immersion. For example, when analyzing collective behavior of baboons [63], often only the 2D trajectories of the animals are considered. When representing the trajectories in most detail (3D) in a virtual environment, the analyst would 
automatically become aware of the circumstance that the animals tend to climb tall trees overnight.

Previous studies have shown a benefit of immersion and VREs for spatial understanding [2], depth perception [1,29], and spatial memory [15]. For example, in soccer analysis, when standing immersed on the virtual soccer field, distances can be estimated more accurately due to stereoscopic perception and familiar distance estimation, since everything is presented according to reality. VR is accompanied by further benefits, as discussed in the previous section. For example, it provides a platform for natural remote collaboration, the entire space in the environment can be used for the display of visualizations, and laws of physics can be bend.

Disclaimer. We do not argue that VR is the one and only solution to minimize the gap between the data world and the real world. For many abstract data types, there is no straightforward mapping to the real world, which allows a visual validation of the real-world context. For instance, when analyzing stock exchange rates, there is no reasonable way to connect the numerical time series data to a physical real-world location. It would not make sense to map analysis results, such as parallel coordinate plots with highlighted clusters, into the virtual duplicate of a trading floor. To apply our approach, a direct connection between the data and the real world is necessary. Moreover, there are many visual analytics applications in which no sufficient data basis exists to reconstruct the real world from the data world and adequately display the analysis results in it. However, there is a wide range of domains in which VR could be used to minimize the discrepancy between the data world and the real world, such as in the emerging fields of collective behavior analysis [8], sports analysis [61], and general geo-spatial data analysis [19]. In the following section, we present an example where we deploy our solution approach for a criminal investigation use case.

\section{Use Case: Crime Scene Investigation}

In this section, we discuss how virtual reality is used in a visual exploration workflow deploying a use case from the field of crime data analysis. To detect differences between the data world and the real world (the curse of visual data exploration), we try to reproduce as much as possible from the real-world scenario in a virtual environment. Analysis results are, additionally, embedded in the reconstruction to be verified and analyzed by a domain expert. We first describe the developed prototype. Subsequently, we focus on the two integrated solution approaches to "break the curse" by reconstructing the real world from the data world and projecting the analysis results into the real world. Afterward, we discuss the role of VR and its benefits in this use case. Last but not least, we provide results from initial expert feedback on the expansion of the screen-based system with VR. 


\subsection{Prototype for 4D Crime Scene Investigation}

The Austrian Security Research Programme KIRAS owned by the Austrian Federal Ministry for Transport, Innovation and Technology (BMVIT).

After a criminal act, police agencies collect all obtainable information to reconstruct the course of events in the minutest detail. Witness videos and surveillance footage are often central sources. The project FLORIDA [7], as well as the EU project VICTORIA [28], focus on facilitating the analysis of large amounts of video data for LEAs. The FLORIDA project is part of a bilateral project of the German BMBF and the Austrian Security Research Programme KIRAS, which is funded by the Austrian Ministry of Transport, Innovation and Technology (BMVIT), and is run by the FFG (Österreichische Forschungsförderungs Gesellschaft). In the scope of these projects, a tool is currently being developed that allows analysts to merge multiple video sources into a single timeline. Therefore, we visualize a $4 \mathrm{D}$ scene (3D space + time) from all input videos by aligning the video sources spatially and temporally. In addition, we enrich the scene with analysis results of semi-automatic object detections from machine learning algorithms. That way, large sets of videos can be inspected simultaneously. Figure 4 shows a sample crime scene (left), an exemplary frame from one of the installed surveillance cameras (center) and a point cloud reconstruction of the crime scene (right). The described use case is based on the multi-camera dataset "IOSB-4D" provided by Pollok [52]. The reconstruction and automatic detections were provided by project partners. Our contribution to the framework is the visualization of all provided information in a (VR) VA environment. In the following, we will explain the framework and its usage.
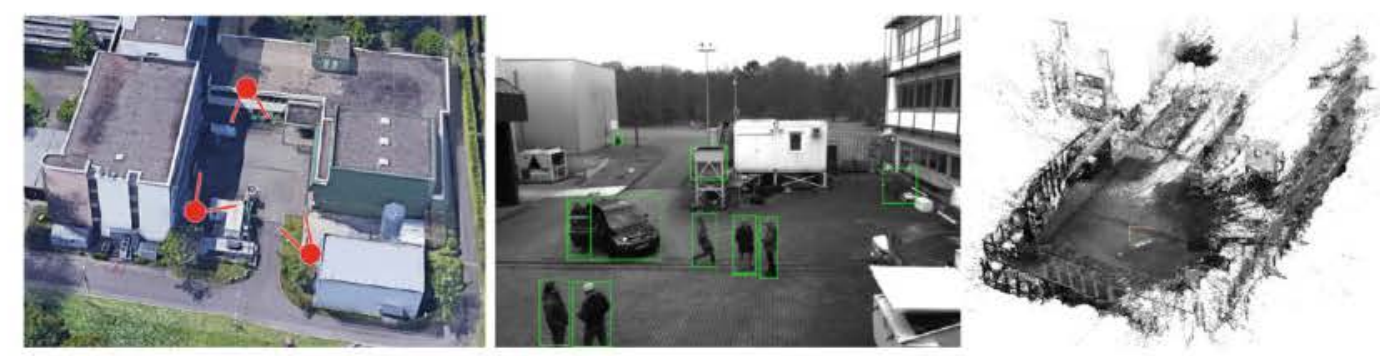

Fig. 4. Crime scene reconstruction. A crime scene (left) is monitored by multiple video cameras (red dots). Each camera provides video footage (center) that can be analyzed with machine learning algorithms (bounding boxes indicate object detections). Using multiple video sources, a 3D point cloud can be reconstructed from the $2 \mathrm{D}$ video streams (right). (Color figure online)

Input Data. The data base for the described analysis is a series of surveillance and witness videos that recorded the same incident from different angles. The sources can be statically installed surveillance cameras or moving hand-held devices, such as cameras or mobile phones. Figure 5 depicts the merging of all video sources into a dynamic $3 \mathrm{D}$ scene that is enriched with automatic detection and analysis results, which are also based on the input videos. 


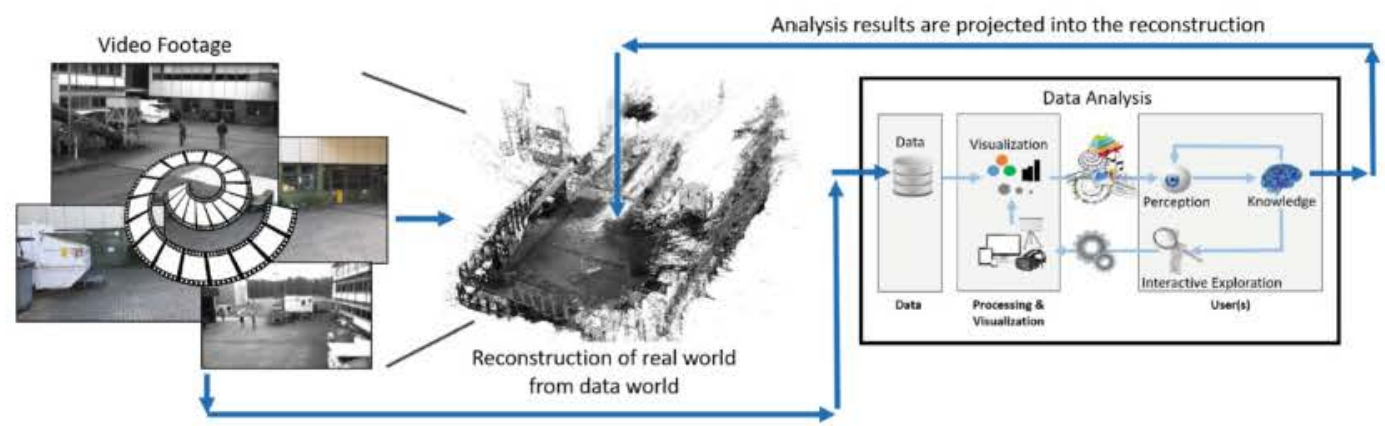

Fig. 5. Multiple video sources are positioned in a 3D scene. With feature matching algorithms, a 3D scene is generated from 2D videos. The origin of each video is registered in this 3D scene. In addition, the videos are analyzed individually, and the results from semi-automatic object detections can be visualized in the 3D scene.

$4 D$ Reconstruction. First, the videos are synchronized temporally by aligning them on a common time axis based on timestamps in the videos and striking events within the videos. In a second step, all video sources are spatially aligned using feature matching algorithms. As a result, for each timestamp on the common time axis, the origins of all video sources are positioned in a $3 \mathrm{D}$ scene. Next, visual features in individual video frames are manually geo-referenced to transform the 3D scene in metric space. For stereoscopic cameras and depth cameras, the videos are transformed into dynamic point clouds as depicted in Fig. 6. Additionally, a static background mesh can be created using a structure from motion approach (see Fig. 7). The credits for the reconstruction go to our project partners from Fraunhofer IOSB.
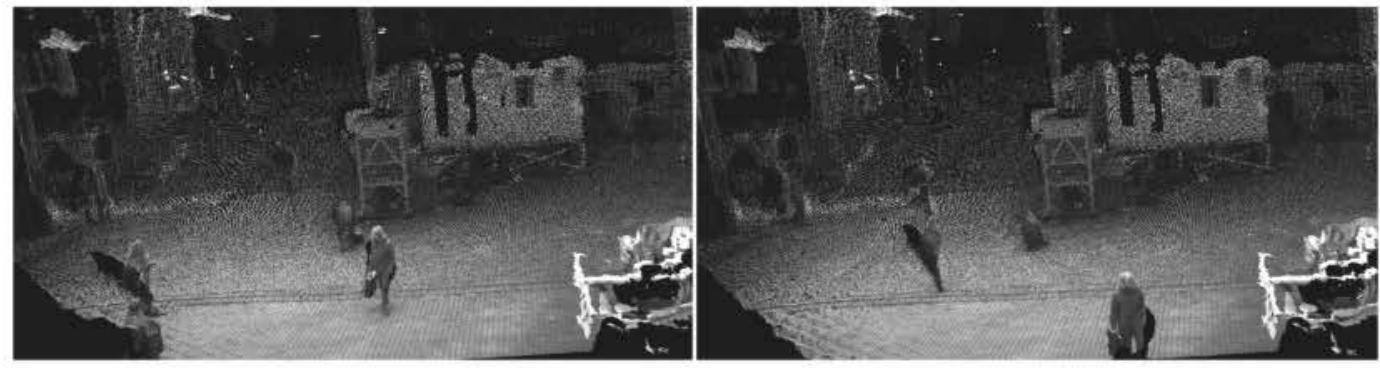

Fig. 6. Dynamic point cloud visualization. Each frame in the source video is displayed as a $3 \mathrm{D}$ point cloud. The $3 \mathrm{D}$ video stream can be played or skipped through by using a timeline slider in the interface.

Video Analysis. The input videos are processed in a semi-automatic feature detection procedure. For each video, objects and persons are detected and tracked for the entire duration of the video. Hereby, pathways of detections are created that can be positioned and visualized in the $3 \mathrm{D}$ scene using triangulation (see Fig. 7). 
Usage of Tool. The main purpose of the developed tool is to support LEA officers in their investigations of crime scenes. The key benefit is the capability of the tool to visualize multiple source videos at once and in the correct spatial and temporal context. The user can explore the large set of witness videos by browsing through the $4 \mathrm{D}$ scene. But the tool is not only suited for the inspection of raw footage videos that are arranged in a $3 \mathrm{D}$ space. It can also be used for more advanced visual analytics procedures. For instance, results of object detection algorithms can be classified in different categories (e.g., person or car) and displayed as heatmaps (Fig. 7, bottom line). This allows the user to quickly grasp the overall distribution of the occurrence of certain objects in the entire scene. The user can refine the visualization by filtering the displayed domain or selecting some classes to display them as multiples of heatmaps. Another example is the analysis of trajectories. Movements of persons and vehicles can be tracked and analyzed. The analysis progress follows the visual analytics pipeline model depicted in Fig. 2. The analyst can select multiple time series to compare, apply operations to them (e.g., clustering, classification, event detection), display intermediate results, refine input parameters, and continue this loop until the results are satisfying. Subsequently, the outcome can be presented in the $4 \mathrm{D}$ scene for verification and inspection.

Figure 7 shows the interface of the $4 \mathrm{D}$ crime scene investigation tool. A timeline at the bottom allows for the temporal navigation within the incident. For spatial navigation, the analyst can fly through the virtual space using a keyboard. A mini-map on the top-right provides an overview of the scene and depicts all registered surveillance cameras in yellow. The original video footage can be inspected by clicking on one of the registered cameras. Detected objects are displayed in the $3 \mathrm{D}$ scene as snippets from the original video material, framed by green bounding boxes.

For the evaluation of evidence footage, it would be possible to dispense from visual analysis and only evaluate the results of object detection algorithms quantitatively. However, visual exploration of video sources combined with automatic detections allows domain experts to identify false classifications, outliers, unrecognized entities, and relationships between subjects that are not visible in the mere metadata from automatic procedures. The merging of all video sources into one $4 \mathrm{D}$ scene allows the analyst to quickly capture the entire data space without having to watch each video separately. Moreover, the spatial and temporal alignment of video sources helps to understand the spatial and temporal connection between different video sources. The presented approach combines the two presented strategies to bridge the gap between the data world and the real world [42]. The real world is reconstructed from the data world by creating a dynamic 3D scene from 2D video footage. Additionally, analysis results (machine learning object detections) are visually projected back into this reconstruction of the real world (see Fig. 4 center). 


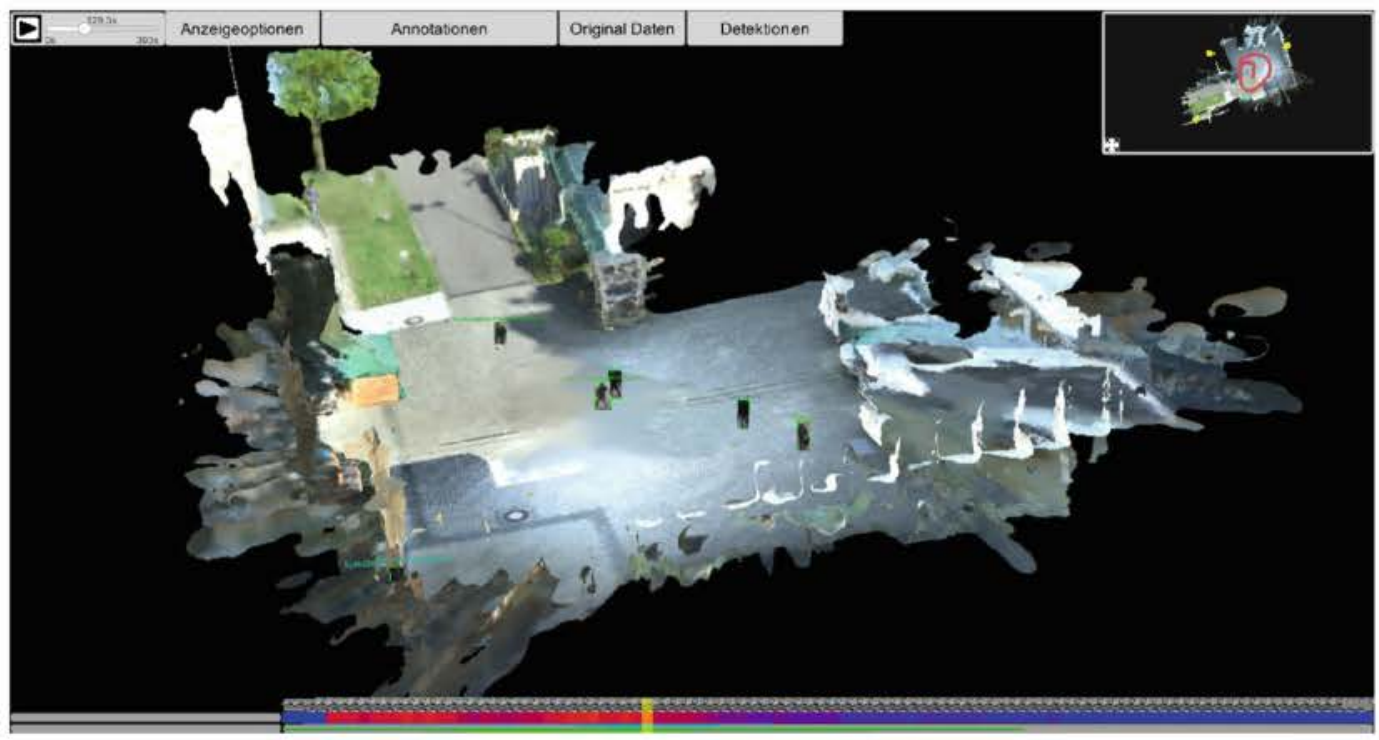

Fig. 7. Reconstruction of the real world from the data world and projection of analysis results. The generated 3D point cloud is converted to a $3 \mathrm{D}$ mesh of the crime scene. Object detections from all video sources can be displayed within the scene (green rectangles with snapshots from videos). (Color figure online)

\subsection{Reconstruction of the Real World}

There is always a discrepancy between the real world and the data world on which the analysis is based on (see Sect. 3). This gap may lead to wrong assumptions about the real-world scenario. In the present case, this may be due to dimensions not considered for analysis or incomplete video sources. For example, the scene may contain spatial or temporal black spots that were not monitored but are highly relevant for the reconstruction of the entire sequence of events.

The reconstruction of the real-world scenario from the data world provides an overview and eases the identification of errors in the data. It may also reveal dimensions that have been neglected in the analysis. If inconsistencies between the data world and the real world are found, they can be resolved, which leads to a minimization of the gap. By merging all available video sources into one $4 \mathrm{D}$ scene, a common frame of reference is established that maximizes the context of each individual video source. For example, when inspecting one video in the $4 \mathrm{D}$ scene, the analyst is aware of all sources that monitor the same area of interest and can switch between multiple videos without having to search the entire pool of footage. This connects all available sources and facilitates the overall analysis. When inspecting the virtual reconstruction of the environment, the analyst subconsciously relates perceived information to known real-world properties. Hence, not modeled dimensions can be identified that have also been neglected in the analysis of individual video sources. For example, a scene may appear unnatural if there is no sky with sun, indicating that weather conditions were not taken into account in the analysis. 


\subsection{Projection of Analysis Results}

The basic idea of this approach is to verify the data of the analysis as well as the analysis outcomes by projecting the results of the analysis into original real-world footage. Displaying analysis results in original footage is less abstract than simply presenting the results without context. For example, when analyzing trajectories of soccer players in a soccer match, it may be more useful to visualize the extracted trajectories directly in the video from which they were extracted than to create an abstract replica of the soccer field for visualization [42]. In the current use case, the $3 \mathrm{D}$ reconstruction of the scene can be used to display spatially and temporally aligned automatic detections. This alignment helps to verify intermediate analysis results, such as automated machine learning outputs. Hereby, falsely classified objects can be easily identified.

Moreover, the projection of analysis results helps to associate detections of the same entity coming from different video sources. For example, if the same person is detected in multiple videos but not matched, this results in multiple individual detections for the same entity. Displaying extracted trajectories that describe the movement of entities can be visually verified. In the automatic extraction process, it often happens that affiliations of trails are mixed up when the trails of two entities cross each other. For example, if two persons go to the same location and move apart again, the trail of the first person is connected to the trail of the second person at the point of encounter and vice versa. Visually, this flaw can be identified easily, but it is hard to detect it in more abstract representations of the analysis results. Additionally, it can happen that the pathway of a single entity is split into multiple segments and is, therefore, considered to be from different entities. By visualizing the segments in the virtual environment, such segmentation can be identified and resolved by merging them into one pathway.

\subsection{Advantages of Deploying Virtual Reality}

Virtual reality allows the analyst to dive into the crime scene and to be fully immersed in the progress of events. The scene can be inspected "from the inside" as if the analyst had been present at the time of the incident. The course of events can be followed in a 3D video of the incident, reconstructed from all available footage. The real world is better replicated since the analyst can naturally walk through the scene. Details, such as the absence of wind or sound, may come to the analyst's mind as the reconstruction is still dissimilar to the known real world.

The reconstruction of the real world from the data world is implicitly improved by changing the way it is observed. The validation of analysis results can be enhanced by augmented depth perception and stereoscopic 3D vision. Distances may be perceived more accurately, allowing a more precise assessment of displayed trajectories. For example, if two waypoints of the trajectory of a person in the scene are far apart, being immersed can help the analyst to classify the respective segment of the trajectory as erroneous. The analyst perceives 
the virtual environment in familiar metric 3D space (distances are measurable in meters), allowing the user to judge properties based on personal experiences from the real world. For instance, it may become obvious that it is not possible for a human being to cover the depicted distance in the given time.

Various studies have shown a positive effect of immersion on spatial memory $[6,9,25]$. In our use case, too, the criminal investigator may benefit from immersion with regard to navigation and orientation capabilities, and it may help the analyst to keep track of suspicious actualities in the scene. This is also fostered by how the analyst can explore the scenario. Instead of having to use tools like mouse and keyboard, the analyst can walk naturally through the virtual environment and focus on the analysis task, not on navigation and possibly complex interaction.

The deployment of VR poses several additional opportunities. Among others, it enables remote collaboration of multiple LEA officers in the shared crime scene reconstruction. This way, a common conversational grounding is established, and the communication is eased by displaying avatars for each participating analyst [4]. The avatars can be used to perform gestures and to call attention to something by pointing at it. Moreover, the VRE offers plenty of space for the installation of infographics, control panels, and interfaces for the overall analysis.

\subsection{Initial Expert Feedback}

The presented tool was developed for LEAs to support them in the investigation of crime scenes. To receive initial expert feedback, we surveyed eleven criminal investigators (CI1-CI11) from the German federal police (Bundespolizei). All invited criminal investigators are employed as police inspectors (degree A10A15) and have between 10 and 35 years of professional experience. All investigators work with video surveillance or video evidence evaluation on a daily basis. First, we demonstrated the capabilities of the tool and gave a tutorial on how to use it. Then, each expert had the opportunity to use both the standard tool on the screen and the VR extension. After that, we conducted a short interview with each participant to obtain qualitative feedback on our approach. The interview consisted of three parts. First, we addressed the crime scene reconstruction. Secondly, we put the focus on the tool's capability to display analysis results, such as object and person detections. Thirdly, we discussed the use of VR and the challenges and benefits it brings.

The results of our initial evaluation are very promising. Investigators acknowledge its potential to be deployed in the fight against crime, among others as a supporting tool for surveillance tasks, recon missions, and digital forensics. VR, in particular, was perceived as having huge potential in this specific domain. VR was even described as a quantum leap forward in criminal investigation by one of the interviewees.

Reconstruction of Crime Scene. The reconstruction of the crime scene is perceived as very helpful for investigators to re-enact the crime by providing a visual basis for imagination (CI7). Image-based reconstruction from evidence footage is 
not only faster, cheaper and more flexible than advanced laser scans of the scene in the retrospective forensic procedure, as it is current state-of-the-art in this domain, but also provides a snapshot of the environment from the time of the incident (CI5). Thus, situational conditions which were present at the time of the incident but changed during or shortly after the incident would not be present in a post-event recording of the environment (e.g., when the scene is scanned with lasers after the incident took place). Moreover, the detailed reconstruction of the crime scene puts all video sources into context, promotes orientation, and helps video analysts to put multiple video sources into a temporal and spatial relation (CI5, CI6). Another benefit mentioned was the versatility of the approach. Analysts do not have to physically go to the crime scene but can inspect it from anywhere (CI9). This may also be used in recon missions to gain orientation in an unknown environment before police forces are deployed. As opposed to watching video sequences from the environment, a 3D reconstruction improves spatial understanding (CI5, CI9, CI10). Currently, digital forensics makes use of $360^{\circ}$ photo spheres to record characteristics of a crime scene. According to one criminal investigator interviewed, distances and sizes are better perceivable in a 3D scene (P7) than in image spheres or videos. Many of the LEA officers saw the need for a more photo-realistic reconstruction and expect great benefits (CI1-CI4, CI6, CI9, CI10). With advancing technological progress, we suggest that the quality of image-based reconstruction will improve significantly shortly and, therewith, minimize this constraint.

Embedding of Analysis Results. A major benefit expressed by the interviewees was that insights from all video sources are merged into one common scene. Not only are the cameras referenced locally in the scene, but also are the detections of their footage set into a temporal and spatial relation. This makes it easier for the investigators to get an overview of the scene and to re-enact the course of events (CI1-CI3, CI8). The reconstruction in combination with automatic detections from the video footage sets all analysis results into context and creates a "big picture" of the scene (CI7). The detections themselves lead the investigators to interesting and important events and characteristics in the huge pool of evidence videos (CI4, CI6). Often, videos are manually inspected, frame by frame, for hours in order to detect wanted persons or vehicles. The automated approach draws investigators' attention to areas in a video that contain movement or even certain types of detections (persons, faces/identities). Moreover, it facilitates the combined inspection of multiple video sources. For example, it allows to easily identify which cameras monitored which area or person at which time (CI4, CI6). Furthermore, the system can be used to verify automatically generated information, such as detections of persons and movement trajectories. When visualizing analysis results, the analyst can validate the information displayed by relating it to the respective context (environment and detections from other cameras) and real-world experiences (CI9). Moreover, it would be beneficial to ease the mental mapping from the data world to the real world for the display of automated detections, if they would be visualized more realistically (CI10). 
For instance, detections of persons walking in the scene should be displayed as $3 \mathrm{D}$ models instead of image snippets moving around.

The Deployment of $V R$. The interviewed investigators mentioned a strong and clear benefit of VR with regard to its capability to project the analyst into the scene. According to one criminal investigator, VR allows to "dive deeper into the crime scene" as "one forgets the physical environment" (CI8). Thus, the 3D scene in VR is more comprehensible, and spatial relations are easier to understand than when inspecting the scene on a screen (CI1-CI3, CI10). Distances can be interpreted more naturally and intuitively (CI1-CI3). As further benefits and future areas of application, the experts mentioned its potential for remote collaboration and mission training in unknown areas (CI1, CI3). As for drawbacks, the investigators mentioned a high learning curve for the new medium and difficulties with the new interaction techniques with controllers (CI9, CI10). Moreover, they saw possible limitations in the high acquisition costs for the required equipment. We argue that this constraint is just a matter of time as it can be expected that prices are going down since VR devices become more and more established.

\section{Discussion}

We have analyzed the applicability of VR for breaking the curse of visual data exploration on a theoretical level. We first showed the theoretical equality of VR to conventional mediums by going step by step through a VA pipeline model. Thereby, we discussed on what properties a VRE has to fulfill to keep up with a monitor screen and identified characteristics of VR that can pose a benefit. Subsequently, we put the focus on VR properties that can help to break the curse of visual data exploration and demonstrated its feasibility in a use case. However, the approach from the use case cannot be applied to any analysis case. For instance, in order to reconstruct the real world from the data world, there must be an analysis scenario that has an unused visualizable context (e.g., a geo-spatial context) and enough data to actually perform a reconstruction. The strategy described is quite costly, and the potential benefit should be estimated in advance. In some cases, it may be sufficient to rely on alternative strategies to verify the accordance of the data world and the real world for the respective analysis. For example, projecting analysis results back into less abstract data can already help to detect misclassifications or discrepancies in the data (e.g., automatic object detections in videos are overplotted in the original videos). For more abstract data, such as stock market developments, the curse of visual data exploration must still be considered, but assessed formally, for instance, with a detailed influencing factor analysis. VR should only be used if the thereby introduced medium has a significant benefit compared to another medium. For instance, in geo-spatial visualizations, it may be useful to deploy VR due to its capability to project the analyst into the scene, which increases immersion and allows the analyst to inspect the scene naturally. 
Currently, state-of-the-art VR technology is placed at a disadvantage because of several drawbacks. Hardware constraints - for instance low resolution or high latency - can introduce physical or psychological phenomena, such as motion sickness or excessive cognitive load. Users are more familiar with non-VR environments, which could affect their overall VR performance. Moreover, many tasks, visualizations, interaction methods, etc. were optimized for conventional desktop PC setups while interaction methods for VR have not yet been sufficiently studied. However, we live in a dynamic era of rapidly evolving technologies, and technological progress may eradicate many of the factors mentioned above shortly, e.g., by providing higher resolution, photo-realistic rendering or advanced interaction technology.

When it comes to the choice of the medium used to display information, it is all about optimizing the visual analytics procedure. According to Chen and Golan [13], this can be seen as an optimization of the cost-benefit ratio. Chen et al. [12] specifically target the cost-benefit analysis for visualizations in VREs. As pointed out in previous work [42], it is important that the analyst can verify the applicability of knowledge generated in the VA process to the investigated real-world scenario. We have demonstrated in the previous sections, how VR can aid such a verification process. However, it depends on the given analysis scenario how the cost-benefit ratio changes when switching from a display-based medium to VR. We argue that the decision to use a VRE as a design space for visualizations must be justified by improvements induced by VR. If the costbenefit ratio is not affected, we consider it advisable to stick to other mediums.

\section{Conclusion}

In this paper, we identified seven dimensions which can be used to compare visualizations presented on different mediums. More precisely, we compared the mediums by means of individual characteristics that are substantial in the visual analytics pipeline. When comparing a generic VR medium with conventional mediums on a conceptional level, a theoretical equivalence of the mediums becomes apparent. Of course, this state will realistically never be fully reached. However, VREs have much potential and can be beneficial in several domains. When choosing the most suitable medium for a specific visualization task, the cost-benefit ratio is a key factor which needs to be considered. We expect technological progress to eliminate some of the current disadvantages of VR in future, which may minimize the "cost-side" and in turn strengthen the "benefit-side" of the cost-benefit equation, providing the basis for an extensive application of VR as a viable medium for presenting and exploring data. We presented a use case showing the potential benefit of VR to break the curse of visual data exploration. In a suitable analysis scenario, real-world circumstances to be analyzed can be reconstructed and enriched with intermediate analysis results. By perceiving the reflection of the real world naturally and with all available contextual information, the analyst may be able to identify discrepancies between reconstruction and reality, such as neglected dimensions that are relevant to the knowledge generation process. 


\section{References}

1. Arms, L., Cook, D., Cruz-Neira, C.: The benefits of statistical visualization in an immersive environment. In: 1999 Proceedings of the IEEE Virtual Reality, pp. 88-95. IEEE (2003). https://doi.org/10.1109/vr.1999.756938

2. Bach, B., Sicat, R., Beyer, J., Cordeil, M., Pfister, H.: The hologram in my hand: how effective is interactive exploration of $3 \mathrm{D}$ visualizations in immersive tangible augmented reality? IEEE Trans. Visual Comput. Graphics 24(1), 457-467 (2018). https://doi.org/10.1109/TVCG.2017.2745941

3. Benford, S., et al.: Coping with uncertainty in a location-based game. IEEE Pervasive Comput. 2(3), 34-41 (2003)

4. Benford, S., Bowers, J., Fahlén, L.E., Greenhalgh, C., Snowdon, D.: User embodiment in collaborative virtual environments. In: Proceedings of the SIGCHI Conference on Human Factors in Computing Systems, CHI 1995, Denver, Colorado, USA, 7-11 May 1995. pp. 242-249 (1995). https://doi.org/10.1145/223904.223935

5. Bidarra, R., Gambon, D., Kooij, R., Nagel, D., Schutjes, M., Tziouvara, I.: Gaming at the dentist's - serious game design for pain and discomfort distraction. In: Schouten, B., Fedtke, S., Bekker, T., Schijven, M., Gekker, A. (eds.) Games for Health, pp. 207-215. Springer, Wiesbaden (2013). https://doi.org/10.1007/978-3658-02897-8_16

6. Bliss, J.P., Tidwell, P.D.: The effectiveness of virtual reality for administering spatial navigation training to police officers. In: Proceedings of the Human Factors and Ergonomics Society Annual Meeting, vol. 39, no. 14, p. 936 (2012). https:// doi.org/10.1177/154193129503901412

7. BMBF: FLORIDA - Flexibles, teilautomatisiertes Analysesystem zur Auswertung von Videomassendaten. http://www.florida-project.de/. Accessed 14 July 2019

8. Buchmuller, J., Cakmak, E., Keim, D.A., Jackle, D., Brandes, U.: MotionRugs: visualizing collective trends in space and time. IEEE Trans. Visual Comput. Graphics 25(1), 76-86 (2018). https://doi.org/10.1109/tvcg.2018.2865049

9. Cánovas, R., León, I., Roldán, M.D., Astur, R., Cimadevilla, J.M.: Virtual reality tasks disclose spatial memory alterations in fibromyalgia. Rheumatology (Oxford, England) 48(10), 1273-1278 (2009). https://doi.org/10.1093/ rheumatology $/$ kep218

10. Card, S.K., Mackinlay, J.D., Shneiderman, B.: Readings in Information Visualization: Using Vision to Think (Interactive Technologies). Morgan Kaufmann Publishers Inc., San Francisco (1999). https://doi.org/10.1234/12345678

11. Chandola, V., Banerjee, A., Kumar, V.: Anomaly detection: a survey. AMC Comput. Surv. (CSUR) 41(3), 15 (2009). https://doi.org/10.1145/1541880.1541882

12. Chen, M., Gaither, K., John, N.W., McCann, B.: Cost-benefit analysis of visualization in virtual environments. IEEE Trans. Visual Comput. Graphics 25(1), 32-42 (2018). http://arxiv.org/abs/1802.09012

13. Chen, M., Golan, A.: What may visualization processes optimize? IEEE Trans. Visual Comput. Graphics 22(12), 2619-2632 (2016). https://doi.org/10.1109/ TVCG.2015.2513410

14. Chi, E.H.: A taxonomy of visualization techniques using the data state reference model. In: IEEE Symposium on Information Visualization 2000 (INFOVIS 2000), Salt Lake City, Utah, USA, 9-10 October 2000, pp. 69-75 (2000). https://doi.org/ 10.1109/INFVIS.2000.885092 
15. Cockburn, A., McKenzie, B.: Evaluating the effectiveness of spatial memory in 2D and 3D physical and virtual environments. In: Proceedings of the SIGCHI Conference on Human Factors in Computing Systems Changing our World, Changing Ourselves - CHI 2002, p. 203 (2002). https://doi.org/10.1145/503376.503413

16. Cruz-Neira, C., Sandin, D.J., DeFanti, T.A.: Surround-screen projection-based virtual reality: the design and implementation of the CAVE. In: The Conference on Computer Graphics and Interactive Techniques (SIGGRAPH 1993), pp. 135-142. ACM (1993). https://doi.org/10.1145/166117.166134

17. Cummings, J.J., Bailenson, J.N., Fidler, M.J.: How immersive is enough? A foundation for a meta-analysis of the effect of immersive technology on measured presence. In: Proceedings of the International Society for Presence Research Conference, vol. 19, no. 2, pp. 272-309 (2012). https://doi.org/10.1080/15213269.2015.1015740

18. Bowman, D.A., McMahan, R.P.: Virtual reality: how much immersion is enough? Computer 40(7), 36-43 (2007). https://doi.org/10.1109/MC.2007.257

19. De Smith, M.J., Goodchild, M.F., Longley, P.: Geospatial Analysis: A Comprehensive Guide to Principles, Techniques, and Software Tools. Troubador Publishing Ltd., Leicester City (2007). https://doi.org/10.1111/j.1467-9671.2008.01122.x

20. Dictionary: Reality: Dictionary. http://www.dictionary.com/browse/reality. Accessed 14 July 2019

21. Dictionary: Virtual: Dictionary. http://www.dictionary.com/browse/virtual. Accessed 14 July 2019

22. Dasgupta, D., Forrest, S.: Novelty detection in time series data using ideas from immunology. In: Proceedings of the International Conference on Intelligent Systems, pp. 82-87 (1996). http://citeseerx.ist.psu.edu/viewdoc/summary?doi=10.1. $1.50 .9949 \mathrm{~d}$

23. Djorgovski, S.G., Donalek, C., Lombeyda, S., Davidoff, S., Amori, M.: Immersive and collaborative data visualization and analytics using virtual reality. In: American Geophysical Union, Fall Meeting 2018, pp. 609-614 (2018). https://doi.org/ 10.1109/BigData.2014.7004282

24. Dos Santos, S., Brodlie, K.: Gaining understanding of multivariate and multidimensional data through visualization. Comput. Graph. (Pergamon) 28(3), 311-325 (2004). https://doi.org/10.1016/j.cag.2004.03.013

25. Dünser, A., Steinbügl, K., Kaufmann, H., Glück, J.: Virtual and augmented reality as spatial ability training tools. In: Proceedings of the ACM SIGCHI New Zealand Chapter's International Conference on Computer-Human Interaction Design Centered HCI - CHINZ 2006, pp. 125-132. ACM (2006). https://doi.org/10.1145/ 1152760.1152776

26. Ellis, S.R.: What are virtual twins? IEEE Comput. Graphics Appl. 14(1), 17-22 (1994). https://www.wisegeek.com/what-are-virtual-twins.htm\#didyouknowout

27. Erra, U., Malandrino, D., Pepe, L.: Virtual reality interfaces for interacting with three-dimensional graphs. Int. J. Hum. Comput. Interact. 35(1), 75-88 (2019). https://doi.org/10.1080/10447318.2018.1429061

28. EU: VICTORIA - Video Analysis for Investigation of Criminal and Terrorist Activities. https://www.victoria-project.eu/. Accessed 14 July 2019

29. Filho, J.A.W., Dal, C., Freitas, S., Rey, M.F., Freitas, C.M.D.S.: Immersive visualization of abstract information: an evaluation on dimensionally-reduced data scatterplots automatic construction of large readability corpora view project blind guardian view project immersive visualization of abstract information: an evaluation In: 2018 IEEE Conference on Virtual Reality and 3D User Interfaces (VR), pp. 483-490 (2018). https://doi.org/10.1109/VR.2018.8447558 
30. Fluke, C.J., Barnes, D.G.: The ultimate display. Multimedia: from Wagner to virtual reality, pp. 506-508 (2016). https://arxiv.org/abs/1601.03459

31. Gershon, N.D.: Visualization of an imperfect world. IEEE Comput. Graphics Appl. 18(4), 43-45 (1998). https://doi.org/10.1109/38.689662

32. Google: Google VR Cardboard. https://www.google.com/get/cardboard. Accessed 14 July 2019

33. Gruchalla, K.: Immersive well-path editing: investigating the added value of immersion. In: IEEE Virtual Reality Conference 2004, pp. 157-164 (2004). https://doi. org/10.1109/VR.2004.42

34. Gutiérrez, F., et al.: The impact of the degree of immersion upon learning performance in virtual reality simulations for medical education. J. Invest. Med. 55, S91 (2007). https://doi.org/10.1097/00042871-200701010-00099

35. Haber, R.B., Mcnabb, D.A.: Visualization ldioms: a conceptual model for scientific visualization systems. Vis. Sci. Comput. 74, 93 (1990)

36. Hodge, V., Austin, J.: A survey of outlier detection methodologies. Artif. Intell. Rev. 22(2), 85-126 (2004). https://doi.org/10.1007/s10462-004-4304-y

37. He, L., Guayaquil-Sosa, A., Mcgraw, T.: Medical image atlas interaction in virtual reality. In: Proceedings of Immersive Analytics Workshop at VIS (2017)

38. HTC: Vive Controller. https://vive.com/eu/accessory/controller/. Accessed 14 July 2019

39. Huang, B., Jiang, B., Li, H.: An integration of GIS, virtual reality and the Internet for visualization, analysis and exploration of spatial data. Int. J. Geogr. Inf. Sci. 15(5), 439-456 (2001). https://doi.org/10.1080/13658810110046574

40. Ijsselsteijn, W., Riva, G.: Being there: the experience of presence in mediated environments, p. 14. In: Emerging Communication: Studies on New Technologies and Practices in Communication. IOS Press, Netherlands (2003). citeulike-articleid: 4444927

41. Kim, W.S.: Computer vision assisted virtual reality calibration. IEEE Trans. Robot. Autom. 15(3), 450-464 (1999)

42. Kraus, M., Weiler, N., Breitkreutz, T., Keim, D., Stein, M.: Breaking the curse of visual data exploration: improving analyses by building bridges between data world and real world. In: Proceedings of the International Joint Conference on Computer Vision, Imaging and Computer Graphics Theory and Applications Volume 3, IVAPP, pp. 19-27. INSTICC, SciTePress (2019). https://doi.org/10. $5220 / 0007257400190027$

43. Krokos, E., Plaisant, C., Varshney, A.: Virtual memory palaces: immersion aids recall. Virtual Reality 23(1) (2019). https://doi.org/10.1007/s10055-018-0346-3

44. Laha, B., Sensharma, K., Schiffbauer, J.D., Bowman, D.A.: Effects of immersion on visual analysis of volume data. IEEE Trans. Visual Comput. Graphics 18(4), 597-606 (2012). https://doi.org/10.1109/TVCG.2012.42

45. Latta, J.N., Oberg, D.J.: A conceptual virtual reality model. IEEE Comput. Graphics Appl. 14(1), 23-29 (1994). https://doi.org/10.1109/38.250915

46. Logitech: Logitech Bridge. https://github.com/Logitech/logi_bridge_sdk. Accessed 14 July 2019

47. MacEachren, A.M., et al.: Visualizing geospatial information uncertainty: what we know and what we need to know. Cartogr. Geogr. Inf. Sci. 32(3), 139-160 (2005). https://doi.org/10.1559/1523040054738936

48. Madole, D., Begault, D.: 3-D Sound for Virtual Reality and Multimedia, vol. 19. Academic Press Professional Inc., San Diego (1995). https://doi.org/10.2307/ 3680997 
49. McGloin, R., Farrar, K., Krcmar, M.: Video games, immersion, and cognitive aggression: does the controller matter? Media Psychol. 16(1), 65-87 (2013). https://doi.org/10.1080/15213269.2012.752428

50. Merino, L., et al.: On the impact of the medium in the effectiveness of 3D software visualizations. In: Proceedings of the 2017 IEEE Working Conference on Software Visualization, October, vol. 2017, pp. 11-21. IEEE (2017). https://doi.org/ 10.1109/VISSOFT.2017.17

51. Niehorster, D.C., Li, L., Lappe, M.: The accuracy and precision of position and orientation tracking in the HTC vive virtual reality system for scientific research. i-Perception 8(3), 2041669517708205 (2017)

52. Pollok, T.: A new multi-camera dataset with surveillance, mobile and stereo cameras for tracking, situation analysis and crime scene investigation applications. In: Proceedings of the International Conference on Video and Image Processing, pp. 171-175 (2018). https://doi.org/10.1145/3301506.3301542

53. Porter, J., Anand, T., Johnson, B., Khan, R.M., Sobel, N.: Brain mechanisms for extracting spatial information from smell. Neuron 47(4), 581-592 (2005). https:// doi.org/10.1016/j.neuron.2005.06.028

54. Prabhat, Forsberg, A.S., Katzourin, M., Wharton, K., Slater, M.: A comparative study of desktop, fishtank, and cave systems for the exploration of volume rendered confocal data sets. IEEE Trans. Visual Comput. Graphics 14(3), 551-563 (2008). https://doi.org/10.1109/TVCG.2007.70433

55. Probst, D., Reymond, J.L.: Exploring DrugBank in virtual reality chemical space. J. Chem. Inf. Model. 58(9), 1731-1735 (2018). https://doi.org/10.1021/acs.jcim. 8 b00402

56. Ragan, E.D., Sowndararajan, A., Kopper, R., Bowman, D.A.: The effects of higher levels of immersion on procedure memorization performance and implications for educational virtual environments. Presence Teleoperators Virtual Environ. 19(6), 527-543 (2010). https://doi.org/10.1162/pres_a_00016

57. Rehm, F., Klawonn, F., Kruse, R.: A novel approach to noise clustering for outlier detection. Soft. Comput. 11(5), 489-494 (2007). https://doi.org/10.1007/s00500006-0112-4

58. Simeone, A.L., Velloso, E., Gellersen, H.: Substitutional reality: using the physical environment to design virtual reality experiences. In: Proceedings of the ACM Conference on Human Factors in Computing Systems, CHI, pp. 3307-3316. ACM (2015). https://doi.org/10.1145/2702123.2702389

59. Skeels, M., Lee, B., Smith, G., Robertson, G.G.: Revealing uncertainty for information visualization. Inf. Vis. 9(1), 70-81 (2010). https://doi.org/10.1057/ivs.2009. 1

60. Slater, M., Wilbur, S.: A framework for immersive virtual environments (FIVE): speculations on the role of presence in virtual environments. Presence Teleoperators Virtual Environ. 6(6), 603-616 (1997). https://doi.org/10.1162/pres.1997.6.6.603

61. Stein, M., et al.: Director's cut: analysis and annotation of soccer matches. IEEE Comput. Graphics Appl. 36(5), 50-60 (2016). ht.tps://doi.org/10.1109/MCG.2016. 102

62. Steuer, J.: Defining virtual reality: dimensions determining telepresence. J. Commun. 42(4), 73-93 (1992). https://doi.org/10.1111/j.1460-2466.1992.tb00812.x

63. Strandburg-Peshkin, A., Farine, D.R., Crofoot, M.C., Couzin, I.D.: Habitat and social factors shape individual decisions and emergent group structure during baboon collective movement. Elife 6, e19505 (2017). https://doi.org/10.7554/eLife. 19505 
64. Suzuki, K., Wakisaka, S., Fujii, N.: Substitutional reality system: a novel. Sci. Rep. 2, 1-9 (2012). https://doi.org/10.1038/srep00459

65. UNDP: United Nations Statistical Commission and Economic Commission for Europe glossary of terms on statistical data editing. https://webgate.ec.europa.eu/ fpfis/mwikis/essvalidserv/images/3/37/UN_editing_glossary.pdf (2018). Accessed 14 July 2019

66. Van der Meijden, O.A.J., Schijven, M.P.: The value of haptic feedback in conventional and robot-assisted minimal invasive surgery and virtual reality training: a current review. Surg. Endosc. 23(6), 1180-1190 (2009). https://doi.org/10.1007/ s00464-008-0298-x

67. Verbree, E., Maren, G.V., Germs, R., Jansen, F., Kraak, M.J.: Interaction in virtual world views-linking 3D GIS with VR. Int. J. Geogr. Inf. Sci. 13(4), 385-396 (1999). https://doi.org/10.1080/136588199241265

68. Wagner Filho, J.A., Freitas, C.M., Nedel, L.: VirtualDesk: a comfortable and efficient immersive information visualization approach. Comput. Graphics Forum 37(3), 415-426 (2018). https://doi.org/10.1111/cgf.13430

69. Walshe, D.G., Lewis, E.J., Kim, S.I., O'Sullivan, K., Wiederhold, B.K.: Exploring the use of computer games and virtual reality in exposure therapy for fear of driving following a motor vehicle accident. CyberPsychol. Behav. 6(3), 329-334 (2003). https://doi.org/10.1089/109493103322011641

70. van Wijk, J.J.: The value of visualization. In: IEEE Visualization Conference, pp. 79-86 (2005). https://doi.org/10.1109/VISUAL.2005.1532781

71. Wills, S., Roecker, S.: Statistics for Soil Survey. http://ncss-tech.github.io/stats_ for_soil_survey/chapters/9_uncertainty/Uncert_val.html\#1_introduction (2017). Accessed 14 July 2019

72. Witmer, B.G., Singer, M.J.: Measuring presence in virtual environments: a presence questionnaire. Presence Teleoperators Virtual Environ. 7(3), 225-240 (1998). https://doi.org/10.1162/105474698565686

73. Zhang, S., et al.: An immersive virtual environment for DT-mri volume visualization applications: a case study. In: Proceedings of the Conference on Visualization, 2001, pp. 437-440. IEEE Computer Society (2001). https://doi.org/10.1109/ VISUAL.2001.964545 